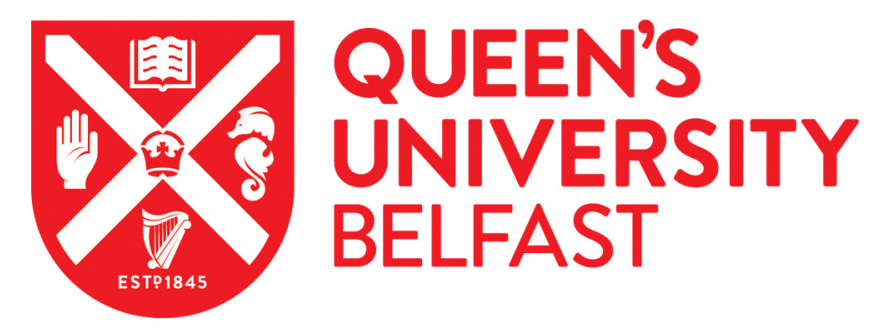

\title{
Trajectories of depression symptoms from pre- to post- deployment: does previous trauma predict symptom increase?
}

Karsoft, K-I., Nielsen, A., Armour, C., Vedtofte, M., \& Andersen, S. (2020). Trajectories of depression symptoms from pre- to post- deployment: does previous trauma predict symptom increase? Journal of Affective Disorders, 266, 120. https://doi.org/10.1016/j.jad.2020.01.112

Published in:

Journal of Affective Disorders

Document Version:

Peer reviewed version

Queen's University Belfast - Research Portal:

Link to publication record in Queen's University Belfast Research Portal

Publisher rights

Copyright 2020 Elsevier.

This manuscript is distributed under a Creative Commons Attribution-NonCommercial-NoDerivs License

(https://creativecommons.org/licenses/by-nc-nd/4.0/), which permits distribution and reproduction for non-commercial purposes, provided the author and source are cited.

\section{General rights}

Copyright for the publications made accessible via the Queen's University Belfast Research Portal is retained by the author(s) and / or other copyright owners and it is a condition of accessing these publications that users recognise and abide by the legal requirements associated with these rights.

Take down policy

The Research Portal is Queen's institutional repository that provides access to Queen's research output. Every effort has been made to ensure that content in the Research Portal does not infringe any person's rights, or applicable UK laws. If you discover content in the Research Portal that you believe breaches copyright or violates any law, please contact openaccess@qub.ac.uk. 


\section{Journal Pre-proof}

Trajectories of depression symptoms from pre- to post- deployment: does previous trauma predict symptom increase?

Karen-Inge Karstoft, Anni B.S Nielsen, Cherie Armour , Mia S Vedtofte, Søren B Andersen

PII: S0165-0327(19)32519-4

DOI: https://doi.org/10.1016/j.jad.2020.01.112

Reference: $\quad$ JAD 11561

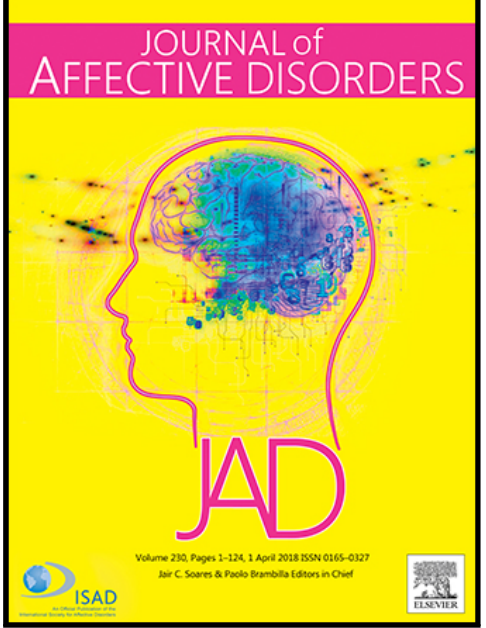

To appear in:

Journal of Affective Disorders

Received date:

17 September 2019

Revised date:

22 December 2019

Accepted date:

20 January 2020

Please cite this article as: Karen-Inge Karstoft, Anni B.S Nielsen, Cherie Armour, Mia S Vedtofte , Søren B Andersen, Trajectories of depression symptoms from pre- to post- deployment: does previous trauma predict symptom increase?, Journal of Affective Disorders (2020), doi: https://doi.org/10.1016/j.jad.2020.01.112

This is a PDF file of an article that has undergone enhancements after acceptance, such as the addition of a cover page and metadata, and formatting for readability, but it is not yet the definitive version of record. This version will undergo additional copyediting, typesetting and review before it is published in its final form, but we are providing this version to give early visibility of the article. Please note that, during the production process, errors may be discovered which could affect the content, and all legal disclaimers that apply to the journal pertain.

(C) 2020 Published by Elsevier B.V. 


\section{Highlights}

- We estimate heterogeneous trajectories of depression symptoms from pre- to 6.5 years postdeployment in 530 Danish soldiers

- In total, 9.4\% experience a dramatic symptom increase from before through 6.5 years after deployment

- Course of depression following deployment is differentially predicted by rank, childhood and adult life trauma, neuroticism, and early post-deployment PTSD-symptoms 


\title{
Trajectories of depression symptoms from pre- to post- deployment: does previous trauma predict symptom increase?
}

\author{
Running title: deployment-related depression and early trauma
}

Karen-Inge Karstofta, b, Anni B.S. Nielsena, c, Cherie Armourd, Mia S. Vedtoftea, Søren B. Andersena

a Research and Knowledge Centre, the Danish Veteran Centre, Ringsted, Denmark.

b Department of Psychology, University of Copenhagen, Denmark.

c The Research Unit and Section of General Practice, Institute of Public Health, University of Copenhagen, Denmark.

d School of Psychology, Queens University Belfast, Belfast, Northern Ireland.

*corresponding author:

Karen-Inge Karstoft

Research and Knowledge Centre, the Danish Veteran Centre

Garnisonen 1

4100 Ringsted

vetc-vic01@mil.dk

$+4572163328$

\section{Abstract}

\section{Background}

A significant minority of individuals experience depression following military deployment. The course of depression symptoms varies over time and across individuals; several factors including combat exposure 
influence depressions incidence and course. Importantly, previous trauma, especially in childhood, have been found increase the risk of post-deployment depression.

\section{Methods}

In a prospective sample of 530 soldiers deployed to Afghanistan in 2009 , we used latent growth mixture modeling (LGMM) to estimate trajectories of depression symptoms from before through 6.5 years after deployment. In a multinomial logistic regression model, we tested if childhood and adult life trauma predicted trajectory membership in combination with combat exposure and neuroticism.

\section{Results}

We identified a large trajectory of few depression symptoms from before through 6.5 years after deployment (Low-stable, 86.5\%), a trajectory with somewhat elevated symptoms (Medium-fluctuating, 4.0\%), and a trajectory with few symptoms before deployment and a steep increase to a severe symptom level 6.5 years after deployment (Low-increasing, 9.4\%). The Low-increasing trajectory was predicted by lower rank and childhood trauma, while the Medium-fluctuating trajectory was predicted by neuroticism, adult life trauma, and post-deployment PTSD symptoms.

\section{Limitations}

Attrition and use of self-report measures for depression and trauma.

\section{Conclusions}

Depression symptoms follow a heterogeneous course from before through 6.5 years after deployment with 9.4\% experiencing symptom increase, resulting in severe symptoms 6.5 years after deployment.

Trajectories are differentially predicted by rank, childhood and adult life trauma as well as neuroticism and PTSD symptoms, illustrating the clinical importance of taking individual differences of symptom course into account.

\section{Keywords}

Depression, military personnel, neuroticism, trauma, prospective studies

\section{Introduction}

Post-deployment depression with or without comorbid PTSD has been found to affect 2-15\% of previously deployed soldiers (Hoge et al., 2004; Reijnen et al., 2015). As such, depression is a relatively common consequence of deployment to war zones. A recent meta-analysis reported increased risk of new-onset 
depression following military combat ( $\mathrm{OR}=1.60$ (95\% $\mathrm{Cl}$ 1.09-2.35); Bonde et al., 2016), however, the studies included in this meta-analysis followed the soldiers for a maximum of 24 months post-deployment, and did not account for individual differences in the course of depression symptoms, which might be crucial (Eeden et al., 2018). To the best of our knowledge, only one study has estimated longitudinal trajectories of depression symptoms following deployment (Sampson et al., 2015), and this study did not include a predeployment assessment of depression symptoms.

In investigating depression trajectories following deployment, Sampson and colleagues assessed 772 previously deployed soldiers for depression symptoms at four time points from approximately seven months after return from deployment until almost four years post deployment (Sampson et al., 2015). Sampson et al.'s study suggested that while most soldiers follow trajectories of resistance (55.9\%) or resilience $(18.7 \%)$ to depression following deployment, a chronic $(12.6 \%)$ as well as a mild increasing (12.8\%) group were also identified. Notably, to date, these conclusions rest on the results of a single post deployment trajectory study and therefore further investigation is warranted.

In the depression literature, childhood trauma has been found to exert a negative impact on the incidence and clinical course of depression in civilian as well as military populations (Bandoli et al., 2017; Jaworska-Andryszewska and Rybakowski, 2019). For example, childhood family problems such as conflicts in the family or parental mental problems as well as physical and sexual abuse has been found to increase the risk of chronic depression in adulthood (Angst et al., 2011; Lindert et al., 2014). One explanation for this link can be found in stress-sensitization theory, which posits that individuals exposed to childhood trauma will develop later depressive symptoms following smaller amounts of preceding stress than individuals without a childhood trauma history (Hammen et al., 2000). Supporting this theory, McLaughlin and colleagues (2010) found, in a national study of 34,653 individuals, that major stressors in adult life were associated with a $27.3 \%$ increase risk of depression among individuals with $3+$ aversive childhood events (ACEs) versus a $14.8 \%$ increased risk among individuals without ACEs.

In a study of 30,436 new soldier recruits, Bandoli et al (2017) found that they were more likely to experience 30-day major depression or generalized anxiety following recent stressful experiences if a history of childhood trauma had been disclosed. Similar findings were reported by Rudenstine and colleagues (2015) who reported an increased risk of post-deployment new-onset depression, but not of post-deployment new-onset PTSD, in soldiers with a history of any childhood trauma. This study did not include measures of combat exposure, but considered deployment in itself to contain potentially significant stressors. Combat exposure was included in another large study that assessed 2,392 soldiers 3 months after deployment to Iraq, and found ACEs to significantly predict post-deployment depression above and beyond 
combat exposure (Cabrera et al., 2007), all pointing towards a significant role of childhood trauma in depression development following deployment, and a possible explanation in stress sensitization theory. However, other perspectives, such as variations of the diathesis-stress model, point to stable individual vulnerability factors such as personality as central mechanisms for the development of depression following stressful life events in adulthood (Colodro-Conde et al., 2018; Kopala-Sibley et al., 2016; Mandelli et al., 2015a). Hence, certain personality factors such as neuroticism might be key in relations between stressful life experiences and depression. Indeed, neuroticism has been suggested as an endophenotype for depression (Goldstein and Klein, 2014; Klein et al., 2011), and has been found to relate to a more chronic course of depression (Mulder, 2002). Therefore, it should be included when studying the link between childhood trauma, deployment and depression.

From the above brief exposition of the literature, it is clear that 1) depression will arise for a significant minority of soldiers following deployment, 2) depression symptoms might follow a heterogeneous course over time in the years following deployment, 3) depression is more frequent and has a more chronic course in individuals subjected to childhood trauma, and 4) childhood trauma as well as neuroticism might interact with deployment stressors to increase the risk of depression. What is lacking is a more detailed perspective on the individual course of depression symptoms in soldiers from before until years after deployment, and how this individual course is predicted from previous trauma in childhood and adult life prior to deployment. Importantly, a pre-deployment depression assessment will enable the disentangling of pre-existing and new-onset depression symptoms following deployment, while homecoming assessments allow us to include combat exposure as an interacting factor and homecoming PTSD symptoms and social support from family, friends and significant other as potentially confounding factors.

Since 2009, a Danish cohort of military personnel deployed to Afghanistan as part of the International Security of Assistance Force (ISAF) has been followed with extensive questionnaires including assessment of depression symptoms before, during, and five times post-deployment with the latest follow up 6.5 years after deployment. Furthermore, previous trauma including trauma in childhood has been assessed as well as demographics, personality, social support and combat exposure. This cohort is therefore perfectly suited for examining the course of depression and the possible risk factors for different trajectories.

The aim of the current study is threefold:

1. To estimate trajectories of depression symptoms from before through 6.5 years after deployment 
2. To evaluate the relative importance of trauma in childhood and adult life predicting these trajectories

3. To test interactions between a) childhood trauma and combat exposure (in line with stresssensitization theory) and b) neuroticism and combat exposure (in line with a diathesis-stress model) in relation to trajectory membership

\section{Material and methods}

\section{Participants}

This study builds on seven waves of data collection on a cohort of Danish military personnel deployed to Afghanistan as part of ISAF in 2009. A total of 743 military personnel were set to deploy in February of 2009, however, 34 did not deploy after all. Further, three died during deployment and were therefore excluded from further analyses. Previous waves have been analyzed in several papers focusing on PTSDsymptomatology, for example Berntsen et al. (2012) and Andersen et al. (2014), however, none have analyzed the presence and course of depression symptoms. This is the first study from this cohort to include the $7^{\text {th }}$ wave of data collection, hence including assessments 5-6 weeks before (T1), during (T2), and five times after deployment (T3: 1-3 weeks after homecoming; T4: 2 months after; T5: 7 months after; T6: 2.5 years after; $77: 6.5$ years after).

For the current analysis, we included participants who had full data on the depression scale at the first assessment (T1) and at two or more additional assessments resulting in a final sample of 530 . We imposed this inclusion criterion since we wanted to include a quadratic term in the model to allow for non-linear variation over time, making three assessments an obvious choice. We compared those included in the analyses to those not included on baseline demographics and found that those included in the analysis were significantly younger, more likely to be male, more likely to be combat soldiers and with fewer previous deployments before this one than their non-included counterparts (see Table 1).

\section{Measures}

Depression symptoms were measured with the Beck Depression Inventory (BDI-II; Beck, 1961). The BDI-II consists of 21 items assessing symptoms of depression within the last two weeks rated on a 0-3 Likert scale. These items are summed to create a total symptom severity score (range 0-63). In our sample across the seven assessments of BDI-II, Cronbach's Alpha varied from 0.83 to 0.94 . To illustrate level of symptom severity in the derived trajectories (see Figure 1), we have plotted the cutoff for severe depression (BDIscore=29; Beck et al., 1988). 
Trauma in childhood and adult life were assessed with the Traumatic Life Events Questionnaire (TLEQ; Kubany et al., 2000). The TLEQ lists 19 traumatic events that might have occurred during a person's life including for example natural disasters, robbery involving a weapon, and physical abuse. Each potential event is responded to with a number indicating how many times that particular trauma has happened to the person. The scale has an upper limit of six, however, indicating that the trauma happened six or more times. Of the listed trauma, three pertain to trauma in childhood: witnessing violence in the family, being physically punished, and sexual abuse. For the purpose of this study, we computed two counts of previous trauma: one of all adult traumas (range 0-96) and one of all childhood traumas (range 0-18).

Neuroticism was assessed with the neuroticism factor of the brief version of NEO Personality Inventory 3 (NEO-PI-3; McCrae and Costa, 2007). Items are rated from 1 (completely disagree) to 5 (completely agree), and 12 items are summed to a total score that expresses neuroticism (range 12-60; Cronbach's Alpha $=0.79$ ).

Combat exposure was assessed at homecoming with the Combat Exposure Scale (CES; Keane et al., 1989), a 7-item measure evaluating exposures such as patrols, being under enemy fire or danger of being injured or killed. Items have five response categories that expresses either frequency (from 1=no to $5=26+/ 51+$ times), duration (from $1=$ never to $5=7+$ months) or proportion (from $0=$ none to $5=76 \%$ or more), and a total score (range 0-41) is constructed by summing weighted item scores, where weights represent the level of exposure.

PTSD symptoms at homecoming were assessed with the PTSD-checklist, civilian version (PCLC; Weathers et al., 1993), a 17-item measure corresponding to the 17 PTSD-items in DSM-IV. Item response options range from 1-5, resulting in a total PCL-score ranging from 17-85. Finally, we used the multidimensional scale of perceived social support (MSPSS; Zimet et al., 1988) to assess perceived social support at homecoming. The MSPSS consists of 12 items rated from 1 to 7 covering three domains: family, friends, and significant other. Further, all items can be summed to compute a total social support scale (range 12-84).

Since the included continuous variables are on different scales with very different ranges, we applied Z-score normalization to all continuous variables to arrive at comparable odds ratios in the regression analysis. 


\section{Analyses}

The main analysis was conducted in Mplus (Muthén and Muthén, 1998). For estimation of trajectories, we applied Latent Growth Mixture Modeling (LGMM), where subpopulations with varying intercepts and slopes are identified in a data driven manner (Jung and Wickrama, 2008). In LGMM, it is assumed that multiple subpopulations exist; however, no assumptions are made about the number of such subpopulations or about their characteristics in form of slope and intercept (Ram and Grimm, 2009). When estimating complex models, it might be necessary to impose constraints on some parameters in order for models to converge. Indeed, we imposed the constraint of no slope variation within identified subpopulations, while the intercept was allowed to vary freely within subpopulations. For model selection, we evaluated the common fit indices, namely the Akaike Information Criteria (AIC; Akaike, 1998), the Bayesian Information Criteria (BIC; Schwarz, 1978) as well as the sample-size adjusted BIC (SSA-BIC). Further, we used the Lo-Mendel-Rubin-likelihood ratio test (LMR-LRT; Lo et al., 2001) and the bootstrap likelihood ratio test (BLRT; McLachlan and Peel, 2000) to statistically compare a model with $\mathrm{K}$ classes to the model with K-1 classes. While we do not base model selection on model entropy, we present it for the reader to evaluate. Finally, in addition to the listed indicators of model fit, we also evaluate model interpretability, theoretical meaningfulness and parsimony to arrive at the model that at once provides close-to-optimal fit of the data without introducing superfluous complexity. For these analyses, we estimate linear as well as quadratic terms to allow for non-linear variation over time.

Evaluation of trajectory membership predictors can be done in several ways (van de Schoot et al., 2017): the most likely class membership variable can be extracted and predictive analyses done post hoc; in a three-step fashion as suggested by Vermunt (2010) or by inclusion of covariates in the model. Each of these methods has limitations and none of the methods can be said to outperform the others in any given data context. Here, given very high entropy of our final model, which minimizes potential bias, we chose to extract the most likely class membership-variables and conduct the multinomial logistic regression analysis post hoc in SPSS. We conduct the regression analysis in two steps: in the first model, we include only pre-deployment variables, whereas in the second model, we include post-deployment variables (combat exposure, PTSD symptoms and social support) and two interactions: childhood trauma and combat exposure (in line with stress sensitization theory) as well as neuroticism and combat exposure (in line with the diathesis-stress model). Previous to variable inclusion, we tested for multicollinearity (and found none).

Exact $p$-values will be presented where relevant to allow for evaluation of statistical significance. The results of the multinomial logistic regression analysis will be presented as odds ratios (ORs) with 95\% confidence intervals (CI). 


\section{Handling of missing data}

As per standard in longitudinal studies and due to the inclusion criteria of $\geq 3$ full data entries on the depression scale, we had missing data at all time points except at the initial assessment. To avoid listwise deletion in the LGMM, we used Full Information Maximum Likelihood (FIML; Enders and Bandalos, 2001). For the post hoc analysis, we included pre-deployment as well as three post-deployment variables. While the rates of missingness were very low for the pre-deployment variables (ranging from $0 \%$ to $3.6 \%$ ), as expected, more individuals had missing data for the post-deployment variables (PCL: $16.6 \%$, Social support: 15.5\%, Combat exposure: $22.5 \%$ ). Little's MCAR-test indicated that data were not missing completely at random (Chi-Square $=120.827, \mathrm{DF}=91, p=.020$ ), hence, listwise deletion of cases with missing data would result in biased estimates (Asendorpf et al., 2014). To avoid this, we applied multiple imputation using predictive mean matching (Horton and Lipsitz, 2001). As per current recommendations (Asendorpf et al., 2014), we created 40 imputed data sets and report the results of the pooled analysis.

\section{Results}

Participants' median age at deployment (T1) was $24\left(25^{\text {th }}\right.$ and $75^{\text {th }}$ percentiles: $\left.22-29\right)$ and $95 \%$ were male (see table 1). Median length of deployment was 193 days $\left(25^{\text {th }}\right.$ and $75^{\text {th }}$ percentiles: $\left.187-197\right)$. One-fourth (25.3\%) have experienced a childhood trauma, whereas most of the participants experienced an adult life trauma prior to deployment (96.2\%). Participant mean on the neuroticism scale was 29.4 (SD=6.2) and 67.6 $(S D=12.2)$ on the social support scale. For BDI, mean and SD at the seven assessments were: T1: 5.1 (5.7), T2: 4.4 (5.4, T3: 4.4 (5.3), T4: 4.8 (6.4), T5: 6.6 (7.6), T6: 7.0 (8.2), and T7: 8.7 (10.7).

Fit indices of estimated models with 1-4 classes can be seen in Table 2. AIC, BIC and SSA-BIC continue to decline with the addition of each new class. LMR-LRT is significant with the addition of class two, but not with the addition of classes three or four, whereas the BLRT is significant with the addition of each new class. The size of the smallest class is reasonable until the addition of class four were the smallest class contains only $1.5 \%$ of the sample. Entropy is high for all models $(>0.90)$. In the literature, it is suggested that model selection is not solely based on LMR-LRT (van de Schoot et al., 2017) and hence, we cannot conclude that the 2-class solution is optimal. Whereas BLRT in simulation-studies seems to perform well for model selection, in empirical studies, it is very often significant regardless of the number of classes. In light of these diverging results, we also base the final model selection on parsimony and class size: while it seems that models with more classes fit the data better, the added fourth class is very small (1.5\%) and seems to be a split of one of the existing three classes. Hence, the four class solution is not very parsimonious, and we therefore select the three-class model as the best representation of our data. 
The three-class model (see Figure 1) consists of a large class with few or no depression symptoms and an almost flat, but significant increase over time (Low-stable; 86.5\%, Intercept (I)=3.90, Slope $(S)=0.04(p=0.048)$, Quadratic term $(Q)=0.00(p=0.495)) ;$ a class with moderately elevated symptoms at baseline and some fluctuation over time (Medium-fluctuating; $4.0 \%, \mathrm{I}=19.00, \mathrm{~S}=-0.43(p=0.146), \mathrm{Q}=0.01$ $(p=0.218)) ;$ and a class with few or no symptoms at baseline and a gradual, significant increase over time, resulting in a very high symptom level 6.5 years after homecoming (Low-increasing; $9.4 \%, \mathrm{l}=3.80, \mathrm{~S}=0.70$ $(p<0.001), Q=-0.00(p=0.037))$.

Results of the post hoc multinomial logistic regression analysis using the Low-stable class as reference can be seen in Table 3. For the model including only pre-deployment variables, we see that membership of the Low-increasing trajectory is predicted by military rank such that higher rank is associated with lower risk of belonging to the Low-increasing trajectory $(\mathrm{OR}=0.25 ; \mathrm{Cl}=0.09-0.67, p=0.006$ ) and by childhood trauma $(\mathrm{OR}=1.53 ; \mathrm{Cl}=1.21-1.94, p<0.001)$. Membership of the Medium-fluctuating trajectory is predicted by neuroticism $(\mathrm{OR}=4.12 ; \mathrm{Cl}=2.26-7.52, p<0.001)$ and trauma in adult life $(\mathrm{OR}=2.00$; $\mathrm{Cl}=1.35-2.98, p<0.001$ ). When adding post-deployment variables, the already identified pre-deployment variables remain significantly related to trajectory membership with only marginal changes in ORs and $p$ values. Further, for the Medium-fluctuating class, higher level of PTSD symptoms is related to trajectory membership (OR=3.14; $\mathrm{Cl}=1.90-5.20, p<0.001)$ while for the Low-increasing class, adult life trauma is associated with trajectory membership with the inclusion of post-deployment variables (OR=1.39; $\mathrm{Cl}=1.10$ 1.91, $p=0.043)$. None of the included interaction terms were significant.

\section{Discussion}

In this analysis of 530 military personnel deployed to Afghanistan with the Danish Defense, we found three trajectories of depression symptoms from before through 6.5 years after deployment: while the majority of the sample (86.5\%) experienced little or no depression symptoms throughout the study period, two symptomatic trajectories emerged. First, a Medium-fluctuating trajectory (4\%) with moderately elevated symptoms pre-deployment, some fluctuation but return to pre-deployment symptom level 6.5 years after homecoming, and second, a Low-increasing trajectory (9.4\%) with few or no symptoms pre-deployment, stability until homecoming and then a gradual increase to a severe depression symptom level 6.5 years post-deployment. Further, we find that rank and childhood trauma are predictive of membership in the Low-increasing group, while neuroticism, adult life trauma, and post-deployment PCL-score are associated with membership of the Medium-fluctuating group. None of the suggested interactions are significant in relation to class membership. 
While no previous studies have estimated depression symptom trajectories in relation to military deployment including a pre-deployment assessment, one previous study estimated depression trajectories from seven months through four years after homecoming (Sampson et al., 2015). While they identified four trajectories, many similarities are apparent across their and our study. First, the majority of participants in the study by Sampson and colleagues belonged to low-symptom trajectories (resilient or resistant, combined $=74.5 \%$, which is also the case in our current study. Sampson and colleagues also identified a trajectory of chronic dysfunction (12.7\%), however, the actual symptom level in this group was relatively moderate, and therefore, not very different from the Medium-fluctuating trajectory identified in our sample. Finally, a smaller group in the Sampson study displayed a gradual increase (Increasing $(\mathrm{mild})=$ $12.8 \%$ ) which is to some degree matched in our study, where a little less than $10 \%$ belong to the Lowincreasing trajectory. However, a very important difference is that in the study by Sampson and colleagues, the symptom level increases only to a very mild level, whereas in our sample, these $9.4 \%$ experience a drastic symptom increase across the study period and display a level of severe depression symptoms 6.5 years after deployment. One factor contributing to this difference might be the timing of assessments: while the study by Sampson and colleagues estimated trajectories starting on average at seven months after deployment, this covers great variation in that the participating soldiers have deployed within the last two years. Hence, the rise in depression symptoms across assessments might not represent responses to deployment. In contrast, the variation around timing of assessment times is small in our cohort, since the entire cohort deployed in the spring of 2009 and had questionnaires distributed to them simultaneously.

Overall, our results are in line with previous findings of depression following military deployment: a 2016 review found that the risk of depression is substantially increased following military combat (Bonde et al., 2016). In our study, most of the cohort remained free of depression symptoms from before through 6.5 year after deployment, however, almost $10 \%$ experienced a dramatic worsening from symptom-free before deployment to a severe level of depression symptoms 6.5 years after deployment. While we cannot make any claims about causality based on our results, and while for example life events and additional deployments in the follow up period might have contributed to this symptom development, it is reasonable to conclude that in our sample, deployment and a rise in depression symptoms coincides for a significant minority.

For the multinomial logistic regression analysis of predictors of class membership, we see that the two symptomatic trajectories in our study are differentially predicted: membership of the Lowincreasing trajectory is predicted by lower military rank and more childhood trauma, while membership of the Medium-fluctuating class is predicted by higher degree of neuroticism and more adult-life trauma. For 
the post-deployment variables, PTSD symptom level at homecoming is strongly associated with membership of the Medium-fluctuating trajectory. These non-overlapping sets of predictors indicate that different mechanisms for depression symptom course might be at play. Sampson et al (2015) also found different factors to predict their depression trajectories, hence, supporting the view that differing courses of depression might be the result of different mechanisms.

That lower rank increases the risk of belonging to the Low-increasing trajectory is in line with previous research of depression following deployment (Ursano et al., 2016). The clear link between childhood trauma and post-deployment increase in depression symptoms (Low-increasing trajectory membership), even with the inclusion of combat exposure, PTSD symptoms and social support at homecoming, supports the notion that early adversity is related to the course of depression (JaworskaAndryszewska and Rybakowski, 2019; Spinhoven et al., 2011). Indeed, the fact that a rise in depression symptoms is seen in the wake of deployment in this trajectory, suggests that childhood trauma might act as a stress sensitizer to increase the risk of depression following deployment (Bandoli et al., 2017; Cabrera et al., 2007; Dorresteijn et al., 2019; Hammen et al., 2000; Rudenstine et al., 2015). However, we found no interaction between childhood trauma and combat exposure, indicating that it might not be the interaction between childhood trauma and combat exposure per se that explains the rise in depression symptoms for this group, but childhood trauma in combination with the general toll of deployment. In addition, it is interesting that previous trauma in adult life did not increase the risk of belonging to the Low-increasing trajectory in the model including only pre-deployment variables, suggesting that the relation between previous trauma and post-deployment depression symptom increase is specific not just to previous trauma, but to previous trauma in childhood (Mandelli et al., 2015b). Of note, adult-life trauma was just exactly significant $(p=0.043)$ in predicting Low-increasing membership in the model including post-deployment variables, suggesting that adult-life trauma might play some role after all in new-onset depression symptoms. However, we suggest that this result is interpreted with caution since the $p$-value is balancing on the limit of statistical significance.

Turning to the predictors of membership of the Medium-fluctuating class, neither childhood trauma nor rank were predictive. Previous adult life trauma was, however, strongly predictive of membership in this class, suggesting that such adult life events are related to depression symptomatology in this cohort of soldiers. Importantly, in the Medium-fluctuating group, moderate depression symptoms were present before deployment, and as such, they should probably not be seen as resulting from the deployment alone. The significance of previous adult life trauma might then be an alternate explanation for their elevated depression symptoms. Further, for the Medium-fluctuating trajectory, we found that 
neuroticism was highly predictive, in line with previous research suggesting neuroticism as an endophenotype for depression (Goldstein and Klein, 2014; Klein et al., 2011). Overall, our results point to neuroticism and trauma in adult life as important for the elevated level of depression symptoms in this group more than combat and deployment. Indeed, we neither found an effect of combat exposure, nor of combat exposure in interaction with neuroticism. While the importance of neuroticism and adult life trauma in depression is in line with previous research (Kopala-Sibley et al., 2016; Mandelli et al., 2015a; Spinhoven et al., 2011), given the retrospective reports of trauma exposure and our limited knowledge of depression symptoms of participants before their enrollment in this study, we cannot make any claims about causality.

Taken together, while nothing conclusive can be said based on this observational study, it seems that the mechanisms of symptom development over time in the two symptom-elevated groups are rather different, with childhood trauma playing a role for symptoms that only start to develop following deployment and not for symptoms fluctuating from before through 6.5 years after deployment. Given the low power to detect potentially small effects of the theory-based interactions, we cannot make strong claims in favor of either of the suggested theoretical mechanisms. However, one important thing should be concluded based on this study: depression symptomatology in the wake of deployment takes more than one form, and simply dichotomizing depression symptoms will lead to neglect of individual differences in symptom course mechanisms. Elucidating such mechanisms and outcomes will pave the way for more individualized intervention strategies.

Finally, we found that neither marital status, gender nor age were significant predictors of trajectory membership, which is somewhat surprising given previous findings (Brewin et al., 2000; Xue et al., 2015). For gender, this might be explained by the small number of women included in this study. This might be further exaggerated in the Medium-fluctuating class, were the small class size gives rise to insecure estimates. However, there is no clear explanation for the missing role of especially age in predicting Low-increasing trajectory membership.

A final relevant discussion when considering post-deployment mental health problems and better phenotyping of such reactions, is the overlap between for example depression outcomes and the more typically studied PTSD outcomes. As mentioned in the introduction, earlier work on this cohort has estimated trajectories of PTSD from before through 2.5 years after deployment (Andersen et al. 2014). When comparing trajectories for the two symptom constellations, we find that 1 ) the PTSD-trajectories provide a more fine-grained picture with six identified trajectories compared to the three depression- 
trajectories and 2) overall, for both symptom constellations, we see that the vast majority of individuals belong to a low-stable trajectory $(78.1 \%$ and $86.6 \%$, respectively), that a proportion starts out with few or no symptoms and then follows a trajectory of steady symptom increase, while the remaining individuals have fluctuating symptoms - with more individual variation in the PTSD symptoms. At the individual level, we find great overlap in trajectory membership, for example, $86 \%$ of those who belong to the Low-stable depression trajectory also belong the Low-stable PTSD-trajectory. Furthermore, we also found a high correlation between the total score for depression and PTSD symptoms (BDI and PCL) at all assessment points (Spearman's rho ranging from $0.68(T 2)-0.82(T 7)$, all p's $<0.001)$, pointing to likely conceptual as well as measurement overlap between the two constructs.

Our study has some limitations. First, our measure of depression symptoms, BDI, has some psychometric limitations, such as poor discriminant validity against anxiety (Richter et al., 1998), which can be assumed to be relatively prevalent in this cohort (Andersen et al., 2014). However, BDI is a commonly used measure, enabling comparison of our results with those of others. Second, our measure of childhood trauma is relatively crude and contains only three items, and contains no information of for example childhood emotional neglect, which has been found to be central in increasing the risk of mental disorders (Nemeroff, 2016). Also, it contains no information about the timing of childhood trauma, which might be very important (Khan et al., 2015). Third, as for all longitudinal studies, we saw some attrition over time, and also, we see important demographic differences between those included and those not included in the study. However, including $75 \%$ of the baseline sample in the analysis of a study running over seven years is acceptable, as long as modern missing data techniques are applied (Gustavson et al., 2012). Fourth, while the logistic regression model points to interesting relationships between covariates and trajectory membership, and while overall classification accuracy of the model is high (88\%), it is based on very high accuracy of classification to the Low-stable class (98.5\%) and limited accuracy of classification to the Medium-fluctuating (33.4\%) and especially the Low-increasing class (8,2\%). Finally, we include postdeployment measures in the second round of our post hoc multinomial logistic regression analysis, which cannot be said to predict trajectory membership, since this assessment is the third time point in the trajectory formation data. However, we believe that selected early post-deployment variables must be included to assess the importance of deployment and early post-deployment characteristics, most importantly combat exposure. Conversely, it is possible that factors following deployment, for example additional traumas in the years after homecoming, affect the development of symptoms. However, given our aim of identifying predictors of trajectory membership, we have not included these factors. 
In spite of these limitations, our findings provide valuable insights on the course of depression from pre-deployment through 6.5 years post-deployment, indicating that symptom course heterogeneity should be taken into account when studying post-deployment depression. Further, our results suggest that rank, personality, and trauma in childhood and adult life are differentially related to individual differences in the course of depression following deployment. From a clinical point of view, assessment of trauma exposure history is important to prospectively identify individuals at risk for development of depression symptoms in the years following a deployment. We suggest that future studies examine in more detail the kind of childhood trauma that are predictive of post-deployment depression symptom onset and negative course.

\section{Acknowledgements}

We are indebted to all military personnel on ISAF7 who have repeatedly and patiently contributed the data for this study.

\section{Funding}

The $6^{\text {th }}$ round of data collection for this study was supported by the TrygFonden.

\section{Conflict of interests}

The authors declare no conflict of interest. 


\section{References}

Akaike, H., 1998. Information Theory and an Extension of the Maximum Likelihood Principle, in: Parzen, E., Tanabe, K., Kitagawa, G. (Eds.), Selected Papers of Hirotugu Akaike, Springer Series in Statistics. Springer New York, New York, NY, pp. 199-213. https://doi.org/10.1007/978-1-4612-1694-0_15

Andersen, S.B., Karstoft, K.-I., Bertelsen, M., Madsen, T., 2014. Latent trajectories of trauma symptoms and resilience: the 3-year longitudinal prospective USPER study of Danish veterans deployed in Afghanistan. J. Clin. Psychiatry. https://doi.org/10.4088/JCP.13m08914

Angst, J., Gamma, A., Rössler, W., Ajdacic, V., Klein, D.N., 2011. Childhood adversity and chronicity of mood disorders. Eur. Arch. Psychiatry Clin. Neurosci. 261, 21-27. https://doi.org/10.1007/s00406-0100120-3

Asendorpf, J.B., van de Schoot, R., Denissen, J.J.A., Hutteman, R., 2014. Reducing bias due to systematic attrition in longitudinal studies: The benefits of multiple imputation. Int. J. Behav. Dev. 38, 453460. https://doi.org/10.1177/0165025414542713

Bandoli, G., Campbell-Sills, L., Kessler, R.C., Heeringa, S.G., Nock, M.K., Rosellini, A.J., Sampson, N.A., Schoenbaum, M., Ursano, R.J., Stein, M.B., 2017. Childhood adversity, adult stress, and the risk of major depression or generalized anxiety disorder in US soldiers: a test of the stress sensitization hypothesis. Psychol. Med. 47, 2379-2392. https://doi.org/10.1017/S0033291717001064

Beck, A.T., 1961. An Inventory for Measuring Depression. Arch. Gen. Psychiatry 4, 561. https://doi.org/10.1001/archpsyc.1961.01710120031004

Beck, A.T., Steer, R.A., Carbin, M.G., 1988. Psychometric properties of the Beck Depression Inventory: Twenty-five years of evaluation. Clin. Psychol. Rev. 8, 77-100. https://doi.org/10.1016/02727358(88)90050-5

Berntsen, D., Johannessen, K.B., Thomsen, Y.D., Bertelsen, M., Hoyle, R.H., Rubin, D.C., 2012. Peace and War: Trajectories of Posttraumatic Stress Disorder Symptoms Before, During, and After Military Deployment in Afghanistan. Psychol. Sci. 23, 1557-1565. https://doi.org/10.1177/0956797612457389

Bonde, J.P., Utzon-Frank, N., Bertelsen, M., Borritz, M., Eller, N.H., Nordentoft, M., Olesen, K., Rod, N.H., Rugulies, R., 2016. Risk of depressive disorder following disasters and military deployment: Systematic review with meta-analysis. Br. J. Psychiatry 208, 330-336. https://doi.org/10.1192/bjp.bp.114.157859

Brewin, C.R., Andrews, B., Valentine, J.D., 2000. Meta-analysis of risk factors for posttraumatic stress disorder in trauma-exposed adults. J. Consult. Clin. Psychol. 68, 748-766.

Cabrera, O.A., Hoge, C.W. Bliese, P.D., Castro, C.A., Messer, S.C., 2007. Childhood Adversity and Combat as Predictors of Depression and Post-Traumatic Stress in Deployed Troops. Am. J. Prev. Med. 33, 7782. https://doi.org/10.1016/j.amepre.2007.03.019

Colodro-Conde, L., Couvey-Duchesne, B., Zhu, G., Coventry, W.L., Byrne, E.M., Gordon, S., Wright, M.J., Montgomery, G.W., Madden, P.A., Ripke, S., Eaves, L.J., Heath, A.C., Wray, N.R., Medland, S.E., Martin, N.G., 2018. A direct test of the diathesis-stress model for depression. Mol. Psychiatry 23, 1590-1596. https://doi.org/10.1038/mp.2017.130

Dorresteijn, S., Gladwin, T.E., Eekhout, I., Vermetten, E., Geuze, E., 2019. Childhood trauma and the role of self-blame on psychological well-being after deployment in male veterans. Eur. J. Psychotraumatology 10, 1558705. https://doi.org/10.1080/20008198.2018.1558705

Eeden, W.A., Hemert, A.M., Carlier, I.V.E., Penninx, B.W., Giltay, E.J., 2018. Severity, course trajectory, and within-person variability of individual symptoms in patients with major depressive disorder. Acta Psychiatr. Scand. acps.12987. https://doi.org/10.1111/acps.12987

Enders, C., Bandalos, D., 2001. The Relative Performance of Full Information Maximum Likelihood Estimation for Missing Data in Structural Equation Models. Struct. Equ. Model. Multidiscip. J. 8, 430-457. https://doi.org/10.1207/S15328007SEM0803_5 
Goldstein, B.L., Klein, D.N., 2014. A review of selected candidate endophenotypes for depression. Clin. Psychol. Rev. 34, 417-427. https://doi.org/10.1016/j.cpr.2014.06.003

Gustavson, K., von Soest, T., Karevold, E., Røysamb, E., 2012. Attrition and generalizability in longitudinal studies: findings from a 15 -year population-based study and a Monte Carlo simulation study. BMC Public Health 12, 918. https://doi.org/10.1186/1471-2458-12-918

Hammen, C., Henry, R., Daley, S.E., 2000. Depression and sensitization to stressors among young women as a function of childhood adversity. J. Consult. Clin. Psychol. 68, 782-787.

Hoge, C.W., Castro, C.A., Messer, S.C., McGurk, D., Cotting, D.I., Koffman, R.L., 2004. Combat Duty in Iraq and Afghanistan, Mental Health Problems, and Barriers to Care. N. Engl. J. Med. 351, 13-22. https://doi.org/10.1056/NEJMoa040603

Horton, N.J., Lipsitz, S.R., 2001. Multiple Imputation in Practice. Am. Stat. 55, 244-254. https://doi.org/10.1198/000313001317098266

Jaworska-Andryszewska, P., Rybakowski, J.K., 2019. Childhood trauma in mood disorders: Neurobiological mechanisms and implications for treatment. Pharmacol. Rep. 71, 112-120. https://doi.org/10.1016/j.pharep.2018.10.004

Jung, T., Wickrama, K.A.S., 2008. An Introduction to Latent Class Growth Analysis and Growth Mixture Modeling. Soc. Personal. Psychol. Compass 2, 302-317. https://doi.org/10.1111/j.17519004.2007.00054.x

Keane, T.M., Fairbank, J.A., Caddell, J.M., Zimering, R.T., Taylor, K.L., Mora, C.A., 1989. Clinical evaluation of a measure to assess combat exposure. Psychol. Assess. J. Consult. Clin. Psychol. 1, 53-55. https://doi.org/10.1037/1040-3590.1.1.53

Khan, A., McCormack, H.C., Bolger, E.A., McGreenery, C.E., Vitaliano, G., Polcari, A., Teicher, M.H., 2015. Childhood Maltreatment, Depression, and Suicidal Ideation: Critical Importance of Parental and Peer Emotional Abuse during Developmental Sensitive Periods in Males and Females. Front. Psychiatry 6, 42. https://doi.org/10.3389/fpsyt.2015.00042

Klein, D.N., Kotov, R., Bufferd, S.J., 2011. Personality and Depression: Explanatory Models and Review of the Evidence. Annu. Rev. Clin. Psychol. 7, 269-295. https://doi.org/10.1146/annurev-clinpsy-032210104540

Kopala-Sibley, D.C., Kotov, R., Bromet, E.J., Carlson, G.A., Danzig, A.P., Black, S.R., Klein, D.N., 2016. Personality diatheses and Hurricane Sandy: effects on post-disaster depression. Psychol. Med. 46, 865-875. https://doi.org/10.1017/S0033291715002378

Kubany, E.S., Leisen, M.B., Kaplan, A.S., Watson, S.B., Haynes, S.N., Owens, J.A., Burns, K., 2000. Development and preliminary validation of a brief broad-spectrum measure of trauma exposure: The Traumatic Life Events Questionnaire. Psychol. Assess. 12, 210-224. https://doi.org/10.1037/1040-3590.12.2.210

Lindert, J., von Ehrenstein, O.S., Grashow, R., Gal, G., Braehler, E., Weisskopf, M.G., 2014. Sexual and physical abuse in childhood is associated with depression and anxiety over the life course: systematic review and meta-analysis. Int. J. Public Health 59, 359-372. https://doi.org/10.1007/s00038-013-0519-5

Lo, Y., Mendell, N.R., Rubin, D.B., 2001. Testing the number of components in a normal mixture. Biometrika 88, 767-778. https://doi.org/10.1093/biomet/88.3.767

Mandelli, L., Nearchou, F.A., Vaiopoulos, C., Stefanis, C.N., Vitoratou, S., Serretti, A., Stefanis, N.C., 2015 a. Neuroticism, social network, stressful life events: Association with mood disorders, depressive symptoms and suicidal ideation in a community sample of women. Psychiatry Res. 226, 38-44. https://doi.org/10.1016/j.psychres.2014.11.001

Mandelli, L., Petrelli, C., Serretti, A., 2015b. The role of specific early trauma in adult depression: A metaanalysis of published literature. Childhood trauma and adult depression. Eur. Psychiatry 30, 665680. https://doi.org/10.1016/j.eurpsy.2015.04.007

McCrae, R.R., Costa, Jr., Paul T., 2007. Brief Versions of the NEO-PI-3. J. Individ. Differ. 28, 116-128. https://doi.org/10.1027/1614-0001.28.3.116 
McLachlan, G., Peel, D., 2000. Finite Mixture Models, 1 edition. ed. Wiley-Interscience, New York.

McLaughlin, K.A., Conron, K.J., Koenen, K.C., Gilman, S.E., 2010. Childhood adversity, adult stressful life events, and risk of past-year psychiatric disorder: a test of the stress sensitization hypothesis in a population-based sample of adults. Psychol. Med. 40, 1647-1658. https://doi.org/10.1017/S0033291709992121

Mulder, R.T., 2002. Personality Pathology and Treatment Outcome in Major Depression: A Review. Am. J. Psychiatry 159, 359-371. https://doi.org/10.1176/appi.ajp.159.3.359

Muthén, L.K., Muthén, B., 1998. Mplus User's Guide. Eigth Edition.

Nemeroff, C.B., 2016. Paradise Lost: The Neurobiological and Clinical Consequences of Child Abuse and Neglect. Neuron 89, 892-909. https://doi.org/10.1016/j.neuron.2016.01.019

Ram, N., Grimm, K.J., 2009. Growth Mixture Modeling: A Method for Identifying Differences in Longitudinal Change Among Unobserved Groups. Int. J. Behav. Dev. 33, 565-576. https://doi.org/10.1177/0165025409343765

Reijnen, A., Rademaker, A.R., Vermetten, E., Geuze, E., 2015. Prevalence of mental health symptoms in Dutch military personnel returning from deployment to Afghanistan: a 2-year longitudinal analysis. Eur. Psychiatry J. Assoc. Eur. Psychiatr. 30, 341-346. https://doi.org/10.1016/j.eurpsy.2014.05.003

Richter, P., Werner, J., Heerlein, A., Kraus, A., Sauer, H., 1998. On the Validity of the Beck Depression Inventory. Psychopathology 31, 160-168. https://doi.org/10.1159/000066239

Rudenstine, S., Cohen, G., Prescott, M., Sampson, L., Liberzon, I., Tamburrino, M., Calabrese, J., Galea, S., 2015. Adverse Childhood Events and the Risk for New-Onset Depression and Post-Traumatic Stress Disorder Among U.S. National Guard Soldiers. Mil. Med. 180, 972-978. https://doi.org/10.7205/MILMED-D-14-00626

Sampson, L., Cohen, G.H., Calabrese, J.R., Fink, D.S., Tamburrino, M., Liberzon, I., Chan, P., Galea, S., 2015. Mental Health Over Time in a Military Sample: The Impact of Alcohol Use Disorder on Trajectories of Psychopathology After Deployment: Trajectories of Mental Health in National Guard Members. J. Trauma. Stress 28, 547-555. https://doi.org/10.1002/jts.22055

Schwarz, G., 1978. Estimating the Dimension of a Model. Ann. Stat. 6, 461-464. https://doi.org/10.1214/aos/1176344136

Spinhoven, P., Elzinga, B.M., Hovens, J.G.F.M., Roelofs, K., van Oppen, P., Zitman, F.G., Penninx, B.W.J.H., 2011. Positive and negative life events and personality traits in predicting course of depression and anxiety: Life events, personality, and affective disorders. Acta Psychiatr. Scand. 124, 462-473. https://doi.org/10.1111/j.1600-0447.2011.01753.x

Ursano, R.J., Wang, J., Ramsawh, H., Russell, D., Benfer, N., Gifford, R.K., Cohen, G.H., Galea, S., Fullerton, C.S., 2016. Post-Traumatic Stress Disorder, Depression, and Binge Drinking in the Reserve Component of the U.S. Armed Forces. Mil. Med. 181, 1287-1293. https://doi.org/10.7205/MILMED-D-15-00445

van de Schoot, R., Sijbrandij, M., Winter, S.D., Depaoli, S., Vermunt, J.K., 2017. The GRoLTS-Checklist: Guidelines for Reporting on Latent Trajectory Studies. Struct. Equ. Model. Multidiscip. J. 24, 451467. https://doi.org/10.1080/10705511.2016.1247646

Vermunt, J.K., 2010. Latent Class Modeling with Covariates: Two Improved Three-Step Approaches. Polit. Anal. 18, 450-469. https://doi.org/10.1093/pan/mpq025

Weathers, F.W., Litz, B.T., Herman, D.S., Huska, J.A., Keane, T.M., 1993. The PTSD Checklist (PCL): Reliability, validity, and diagnostic utility, in: Annual Convention of the International Society for Traumatic Stress Studies, San Antonio, TX. San Antonio, TX.

Xue, C., Ge, Y., Tang, B., Liu, Y., Kang, P., Wang, M., Zhang, L., 2015. A meta-analysis of risk factors for combat-related PTSD among military personnel and veterans. PloS One 10, e0120270. https://doi.org/10.1371/journal.pone.0120270

Zimet, G.D., Dahlem, N.W., Zimet, S.G., Farley, G.K., 1988. The Multidimensional Scale of Perceived Social Support. J. Pers. Assess. 52, 30-41. https://doi.org/10.1207/s15327752jpa5201_2 


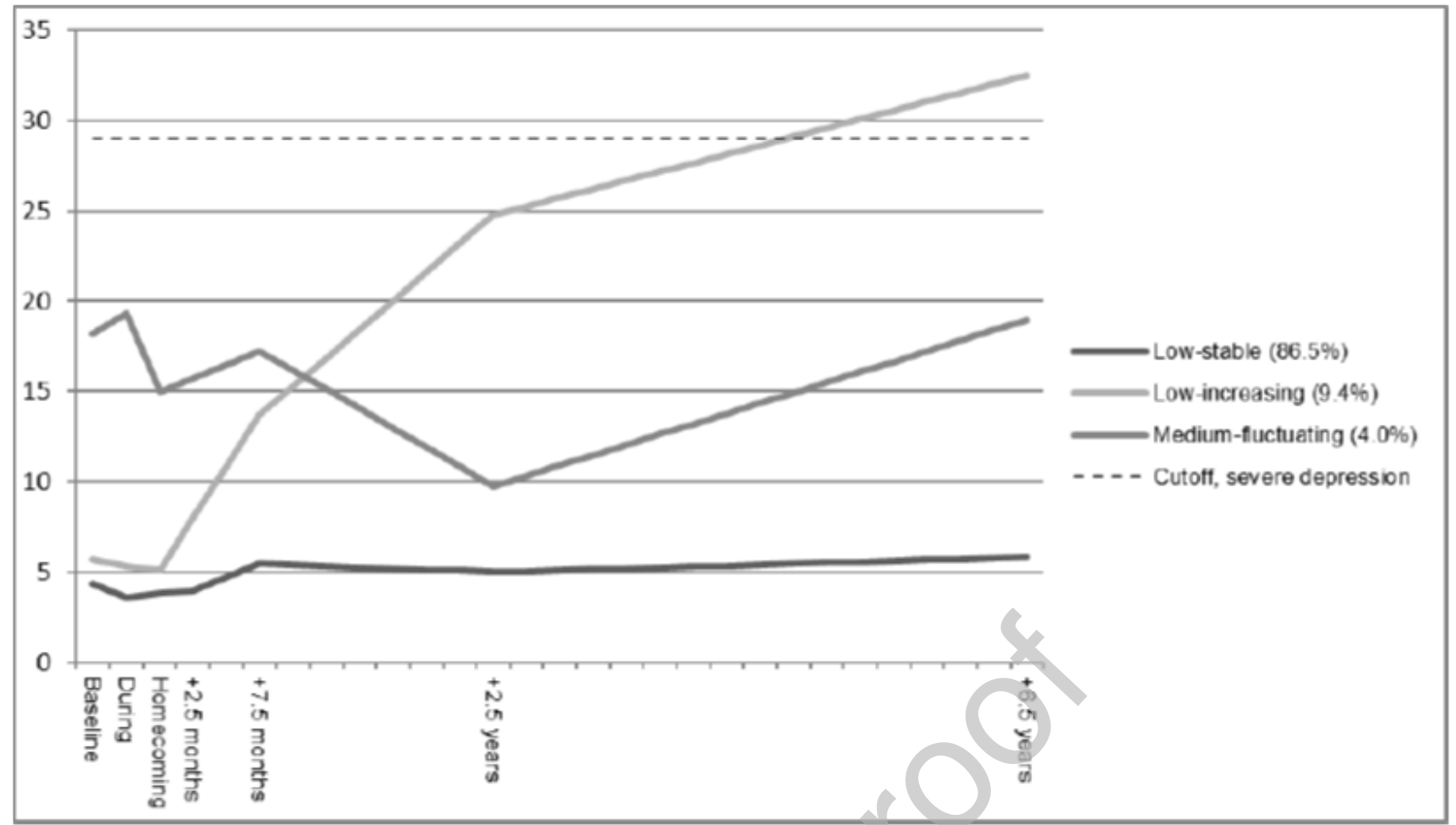

Figure 1. Trajectories of depression symptoms from before through 6.5 years after deployment among Danish military personnel.

Notes: Dotted line represents the cutoff for severe depression as measured on the BDI.

\begin{tabular}{r|ccc} 
& $\begin{array}{c}\text { Included in } \\
\text { sample }\end{array}$ & $\begin{array}{c}\text { Not included in } \\
\text { sample }\end{array}$ & P-value \\
\hline Age at deployment (median $\left(25^{\text {th }}-75^{\text {th }}\right.$ percentile)) & $24(22-29)$ & $29(23-36)$ & $<0.001$ \\
Sex (\% female) & $5.0 \%$ & $9.8 \%$ & 0.022 \\
Deployment no. (median(25 $-75^{\text {th }}$ percentile)) & $1(1-2)$ & $2.3(1-3)$ & $<0.001$ \\
Combat soldier (vs. Different function) & $44 \%$ & $31 \%$ & 0.003 \\
\hline
\end{tabular}

Table 1. Sample demographics stratified to those included and not included in the analysis sample.

Notes: Non-normal continuous variables (age and number of previous deployments) are compared using Mann-

Whitney U test. 


\begin{tabular}{|c|c|c|c|c|c|c|c|}
\hline & $A I C$ & $B I C$ & SSA-BIC & $\begin{array}{c}\text { LMR- } \\
\text { LRT }\end{array}$ & $B L R T$ & Entropy & Smallest class \\
\hline 1 class & 17620 & 17667 & 17632 & $\mathrm{~N} / \mathrm{A}$ & $\mathrm{N} / \mathrm{A}$ & N/A & N/A \\
\hline 2 classes & 17334 & 17399 & 17351 & 0.006 & $<0.001$ & 0.92 & $9.8 \%$ \\
\hline 3 classes & 17251 & 17332 & 17272 & 0.504 & $<0.001$ & 0.93 & $4.0 \%$ \\
\hline 4 classes & 17173 & 17272 & 17199 & 0.113 & $<0.001$ & 0.93 & $1.5 \%$ \\
\hline
\end{tabular}

Table 2. Fit indices of models with 1-4 classes.

Abbreviations: $A I C=$ Akaike information criterion, $B I C=$ Bayesian information criterion, $S S A-B I C=$ Sample-size adjusted $B I C, L M R-L R T=L o-M e n d e l-R u b i n-l i k e l i h o o d$ ratio test, $B L R T=$ the bootstrap likelihood ratio test.

\begin{tabular}{|c|c|c|c|c|}
\hline & \multicolumn{2}{|c|}{$\begin{array}{c}\text { Only pre-deployment } \\
\text { variables }\end{array}$} & \multicolumn{2}{|c|}{$\begin{array}{c}\text { Pre-deployment }+ \text { homecoming } \\
\text { variables }\end{array}$} \\
\hline & Low-increasing & $\begin{array}{l}\text { Medium- } \\
\text { fluctuating }\end{array}$ & Low-increasing & $\begin{array}{l}\text { Medium- } \\
\text { fluctuating }\end{array}$ \\
\hline Gender (ref: male) & $\begin{array}{c}0.65 \\
(0.14-3.09)\end{array}$ & $\begin{array}{c}3.57 \\
(0.82-15.38)\end{array}$ & $\begin{array}{c}0.45 \\
(0.08-2.54)\end{array}$ & $\begin{array}{c}2.22 \\
(0.34-14.49)\end{array}$ \\
\hline Age & $\begin{array}{c}1.07 \\
(0.76-1.51)\end{array}$ & $\begin{array}{c}0.91 \\
(0.46-1.79) \\
\end{array}$ & $\begin{array}{c}1.02 \\
(0.69-1.48)\end{array}$ & $\begin{array}{c}0.84 \\
(0.33-2.18)\end{array}$ \\
\hline $\begin{array}{l}\text { Marital status (ref: in } \\
\text { a relationship) }\end{array}$ & $\begin{array}{c}0.84 \\
(0.43-1.65)\end{array}$ & $\begin{array}{c}1.84 \\
(0.65-5.20)\end{array}$ & $\begin{array}{c}0.93 \\
(0.45-1.86)\end{array}$ & $\begin{array}{c}2.19 \\
(0.58-8.26)\end{array}$ \\
\hline Rank (ref: privates) & $\begin{array}{c}0.25^{* *} \\
(0.09-0.67)\end{array}$ & $\begin{array}{c}2.80 \\
(0.86-5.21)\end{array}$ & $\begin{array}{c}0.26 * * \\
(0.09-0.72)\end{array}$ & $\begin{array}{c}2.23 \\
(0.52-9.43)\end{array}$ \\
\hline Neuroticism & $\begin{array}{c}0.76 \\
(0.54-1.08)\end{array}$ & $\begin{array}{c}4.12 * * * \\
(2.26-7.52)\end{array}$ & $\begin{array}{c}0.72 \\
(0.50-1.04)\end{array}$ & $\begin{array}{c}2.98^{* *} \\
(1.42-6.27)\end{array}$ \\
\hline Childhood trauma & $\begin{array}{c}1.53 * * * \\
(1.21-1.94)\end{array}$ & $\begin{array}{c}0.83 \\
(0.52-1.34)\end{array}$ & $\begin{array}{c}1.43^{* *} \\
(1.11-1.86)\end{array}$ & $\begin{array}{c}0.34 \\
(0.09-1.24) \\
\end{array}$ \\
\hline Adult life trauma & $\begin{array}{c}1.29 \\
(0.97-1.72)\end{array}$ & $\begin{array}{c}2.00 * * * \\
(1.35-2.98)\end{array}$ & $\begin{array}{c}1.39 * \\
(1.01-1.91)\end{array}$ & $\begin{array}{c}2.30 * * \\
(1.36-3.88)\end{array}$ \\
\hline Combat exposure & & & $\begin{array}{c}0.75 \\
(0.46-1.23)\end{array}$ & $\begin{array}{c}0.95 \\
(0.37-2.45)\end{array}$ \\
\hline $\begin{array}{l}\text { Home coming PCL- } \\
\text { score }\end{array}$ & & & $\begin{array}{c}1.26 \\
(0.84-1.89)\end{array}$ & $\begin{array}{c}3.14 * * * \\
(1.90-5.20)\end{array}$ \\
\hline $\begin{array}{l}\text { Home coming social } \\
\text { support }\end{array}$ & & & $\begin{array}{c}1.12 \\
(0.74-1.69)\end{array}$ & $\begin{array}{c}1.41 \\
(0.63-3.14)\end{array}$ \\
\hline $\begin{array}{l}\text { Childhood trauma } x \\
\text { Combat exposure }\end{array}$ & & & $\begin{array}{c}0.93 \\
(0.72-1.21)\end{array}$ & $\begin{array}{c}1.34 \\
(0.43-4.22)\end{array}$ \\
\hline $\begin{array}{l}\text { Neuroticism } \mathrm{x} \\
\text { Combat exposure }\end{array}$ & & & $\begin{array}{c}0.92 \\
(0.63-1.33)\end{array}$ & $\begin{array}{c}0.55 \\
(0.27-1.12)\end{array}$ \\
\hline
\end{tabular}

Table 3. Multinomial logistic regression analysis as implemented post hoc examining trajecoty membership among 530 Danish military personnel followed until 6.5 years after homecoming from international mission.

Notes: The Low-stable trajectory is used as the reference class (=1.00). The left-most column presents results of the multinomial logistic regression including only pre-deployment variables, while the right-most column presents results of the model including variables assessed at home coming. ORs and 95\% Cls are presented. *: $p$-value<0.05; **: $p$ value $<0.01 ; * * *$-value $<0.001$. For exact $p$-values of significant results, see text. 